\title{
Inflammation in uveal melanoma
}

IHG Bronkhorst and MJ Jager

\begin{abstract}
Leukocytic infiltration is a common feature of human cancers, including those that develop in immunoprivileged sites, such as the eye. The infiltration of myeloid and $T$ cells into tumours is part of the host response against cancer. In uveal melanoma, high densities of immune cells seem to be involved in tumour progression, as they are associated with the loss of one chromosome 3 . The nature of this tumour microenvironment might offer therapeutic opportunities.
\end{abstract}

Eye (2013) 27, 217-223; doi:10.1038/eye.2012.253;

published online 14 December 2012

Keywords: uveal melanoma; tumour immunology; macrophages; lymphocytes; cytokines; hypoxia

\section{The link between inflammation and cancer}

Inflammation is so prevalent in malignant neoplasms that it is widely regarded as one of the hallmarks of cancer. ${ }^{1,2}$ Why does this happen? What does inflammation do? Are cancers suppressed or stimulated by the immune response? Can immunity be harnessed to be deployed as a therapeutic tool? How relevant is tumour immunology to uveal melanoma?

For many years, it was assumed that inflammatory cells in cancers reflected antitumour responses. Thanks to advances in identifying different cell subtypes, however, there is growing evidence that inflammation has an important role in the initiation phase of malignancy as well as influencing tumour progression. Inflammation can contribute to angiogenesis, metastasis, antitumour immune responses, and reactions to chemotherapeutic agents.

The seventh hallmark of cancer is defined as cancer-related inflammation, which is characterized by leukocyte infiltration and the presence of soluble mediators, such as cytokines and chemokines. ${ }^{3}$ One would expect that the infiltration of leukocytes into tumours would lead to tumour cell elimination before the tumour becomes clinically apparent; however, this is often not the case. One of the major obstacles may be local immune suppression within the tumour microenvironment. ${ }^{4}$ Immune cells that are able to kill tumour cells in vitro can be inhibited in vivo from killing the malignancy.

In this article, we summarize the current knowledge on inflammation in uveal melanoma, also discussing the scope for further research. Particular emphasis is placed on the characterization of the inflammatory microenvironment.

\section{Uveal melanoma}

Uveal melanoma affects about seven individuals per million per year. Nevertheless, it is the most common primary intraocular malignancy in adults. It originates from the melanocytes in the eye. Uveal melanomas share some features with cutaneous melanomas. For example, the biochemical changes that induce cell proliferation are quite similar in these two malignancies. However, there are major differences between the initiating events in these two kinds of melanoma. For instance, in cutaneous melanoma, BRAF gene mutations are initiators of malignancy, whereas in uveal melanoma, this role is played by mutations in the GNAQ and GNA11 genes. ${ }^{5-9}$ Such mutations activate biochemical pathways that induce cell division.

Ocular melanoma metastasizes hematogeneously. Metastatic disease develops in up to $50 \%$ of patients, usually involving the liver. The average survival time after diagnosis of liver metastasis is about 8 months. ${ }^{10}$ Treatment only rarely prolongs survival, because metastases are highly resistant to most chemotherapeutic agents and because they are not usually resectable. Tumours with a high risk of metastasis can be identified by many techniques.

\section{Prognostic factors}

Uveal melanomas can be classified histologically as spindle, epithelioid, or mixed.
Department of Ophthalmology, Leiden University Medical Center (LUMC), Leiden, The Netherlands

Correspondence: MJ Jager, Department of Ophthalmology, Leiden University Medical Center (LUMC), PO Box 9600, Leiden 2300, The Netherlands Tel: + 3171526 3097; Fax: + 31715248222 . E-mail: m.j.jager@lumc.nl

Received: 25 October 2012 Accepted: 30 October 2012 Published online: 14 December 2012 
Those with epithelioid cells are associated with a worse prognosis. Tumour size (as measured by largest basal diameter and thickness) is an important prognostic parameter. The TNM staging classification combines tumour size, involvement of the ciliary body, and extrascleral extension to categorize uveal melanomas into prognostic groups. ${ }^{11}$ Survival probability cannot be estimated reliably in individual patients on the basis of intraocular tumour size alone. ${ }^{12}$ Damato and co-workers ${ }^{13}$ have therefore developed mathematical methods that integrate clinical and histological predictors with chromosomal abnormalities to enhance prognostication. ${ }^{13}$ Chromosomal markers are common and include chromosome $8 \mathrm{q}$ gain, chromosome $6 \mathrm{p}$ gain, and chromosome 3 loss. ${ }^{14}$ A different approach to prognostication is gene expression profiling. ${ }^{6,15}$ Metastatic death occurs almost exclusively in patients with chromosome 3 loss and/or a class 2 gene expression profile. Other parameters related to poor prognosis include immunological determinants.

\section{Prognostic impact of intratumoral immune infiltrates}

The presence of infiltrating immune cells in and around tumours and their relation with clinical outcome have led to the hypothesis that the immune microenvironment is an important prognostic factor in cancer. ${ }^{16}$ The presence of large numbers of lymphocytes is associated with a good prognosis in many cancer types, such as cutaneous melanoma, non-Hodgkin's lymphoma, non-small-cell lung cancer, and breast cancer (reviewed by Mlecnik et $a l^{17}$ ). In uveal melanoma, however, an inflammatory phenotype is associated with a poor outcome and has been found to be associated with loss of one chromosome $3 .{ }^{18}$ Uveal melanoma may show increased numbers of CD3 + T lymphocytes and CD11b + macrophages. ${ }^{19}$ High densities of inflammatory cells occur more frequently in epithelioid-cell-type tumours, which are especially the tumours with chromosome 3 loss. ${ }^{18}$ To understand the cause of the influx of inflammatory cells, one has to study the composition and function of the immune infiltrate, in the context of microenvironmental factors, such as hypoxia. ${ }^{20}$

\section{Immunology}

The main functions of the immune system are to eliminate pathogens and eradicate developing tumours, while preventing autoreactive responses that are harmful to the host. A complex interplay between immune cells exists. Deregulation of this stimulatory and inhibitory balance is directly associated with many human diseases, which include inflammatory and autoimmune disorders, infection, and cancer (Box 1). The ocular immune system
Box 1 Inflammation and cancer-basic facts (adapted from Grivennikov et $a l^{32}$ )

- Various types of immune and inflammatory cells are frequently present within tumours.

- Immune cells affect malignant cells through the production of cytokines, chemokines, growth factors, prostaglandins, and reactive oxygen and nitrogen species.

- Inflammation may impact every single step of tumourigenesis, from initiation through tumour promotion, all the way to metastatic progression.

- In developing tumours, anti- and protumourigenic immune and inflammatory mechanisms coexist, but if the tumour is not rejected, the protumourigenic effect dominates.

and its role in tumour biology have been intensively studied, leading to the discovery of the ocular immune privilege incorporating the phenomenon of anterior chamber-associated immune deviation. ${ }^{21}$ Ocular immune privilege helps to minimize immunopathological processes, thereby preserving vision.

The inflammatory response associated with regular wound healing is usually self-limiting, and once tissue regeneration is complete, inflammation subsides. During tumour development, cell proliferation results in tissue expansion. Tumours have been referred to as 'wounds that do not heal', ${ }^{22}$ based on the continuous cell renewal and proliferation induced by tumour-associated inflammation. Early and persistent inflammatory responses provide an environment suitable for neoplastic progression: ${ }^{23}$ tumour cells may modulate the functions of surrounding cells to facilitate their own growth, survival, invasion, and metastasis. This interplay between cancer cells and surrounding components opens new possibilities for novel treatments.

\section{Tumour recruitment of immune cells}

Conventional macrophages and dendritic cells have functions such as antigen presentation and T-cell activation, which may be important in providing antitumour immunity. These cells may also produce immunosuppressive cytokines, however, which may suppress tumour immunity.

\section{Macrophages}

Macrophages are involved in tumour development as well as wound healing. Macrophages are monocytes that originate in the bone marrow and differentiate on 
extravasation from the blood circulation. They are recruited to sites of tissue injury, inflammation, or cell proliferation by specific chemokines, such as monocyte chemotactic protein-1. Macrophages themselves are a major source of growth factors and cytokines that profoundly affect other cells in their vicinity. ${ }^{24}$ Tumourassociated macrophages (TAMs) mostly promote tumour growth and may be obligatory for angiogenesis, invasion, and metastasis. A high TAM content generally correlates with poor prognosis. ${ }^{25}$ TAMs come in two kinds: M1 and M2 macrophages. M1 macrophages are activated by IFN $\gamma$ and microbial products, and express high levels of proinflammatory cytokines, major histocompatibility complex molecules, and inducible nitric oxide synthase. M2 macrophages have a low major histocompatibility complex class II and interleukin (IL)-12 expression and show increased expression of the anti-inflammatory cytokine IL-10, scavenger receptor A, and arginase. Cytokines produced by cells in the tumour microenvironment determine whether TAMs become M1 or M2.

Uveal melanoma vary in the amount of CD68+ macrophages they contain. ${ }^{26}$ About $17 \%$ of tumours contain few macrophages, with $32 \%$ having many such cells and $51 \%$ showing moderate numbers. The density of TAMs correlates with metastatic mortality (Figure 1) as well as with female gender, large basal tumour dimensions, epithelioid cell type, heavy pigmentation, and a high microvascular density. In Leiden, we found that the majority of macrophages belong to the M2 type ${ }^{27}$ $($ CD68 + CD163 + ; Figure 2). This is the predominant TAM phenotype in most cancers. ${ }^{28} \mathrm{M} 2$ macrophages have only a low tumouricidal activity, mostly promoting tissue remodelling and angiogenesis. It would be preferable if uveal melanomas contained more classically activated M1-polarized macrophages, which have the

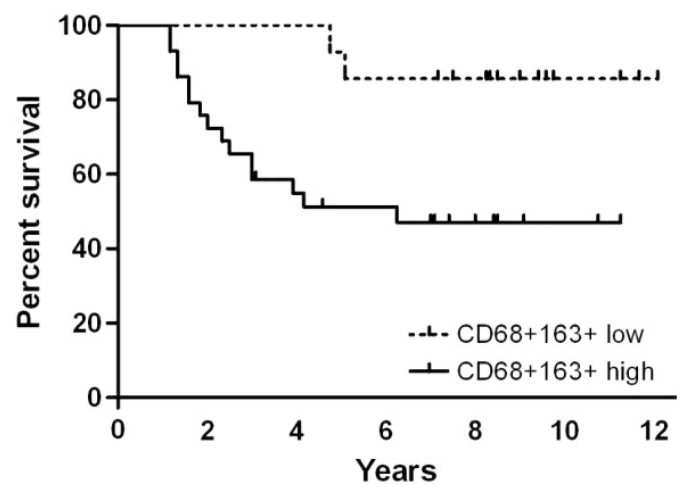

Figure 1 Kaplan-Meier curve showing melanoma-specific survival (update of Bronkhorst et $a l^{27}$ ). Prognosis of mortality due to metastasis was significantly better among patients with low CD68 + CD163 + staining. potential to exhibit antitumour activity and to elicit tumour destruction.

The importance of M2 macrophages in intraocular tumours was investigated using a melanoma mouse model, which showed that the innate immune system can dramatically affect the degree of tumour growth. ${ }^{29}$ When neoplastic cells were implanted into the anterior chamber of mice, tumours developed in both young and old mice. However, when macrophages were depleted at the time of implantation, tumours no longer developed in the eyes of old mice. This is because their growth depended on the presence of protumour M2 macrophages, which are more prevalent in old mice than in young mice. It is necessary to determine whether the findings from mouse tumour models are relevant to humans. The density of macrophages in human uveal melanoma correlates with the density of blood vessels, supporting the hypothesis that macrophages have a role in intratumoral angiogenesis. ${ }^{26,27,30}$

\section{T cells}

Besides TAMs, the other immune cells that are frequently found in the tumour microenvironment are lymphocytes (T cells). Mature T cells are classified according to their effector functions as: (1) CD8 + cytotoxic T cells (CTLs); (2) CD4 + helper T (Th) cells, which include Th1, Th2, and Th17; and (3) T regulatory (Treg) cells. Importantly, $\mathrm{T}$ cells can exert both tumour-suppressive and -promoting effects, as determined by their effector functions. ${ }^{31}$ As reviewed by Grivennikov et al, ${ }^{32}$ increased numbers of T cells, specifically, activated CTLs, correlate with better survival in some cancers, including cutaneous melanoma. However, there is also evidence that many of the T-cell subsets found in solid tumours, such as uveal melanoma, are involved in tumour promotion, progression, and metastasis. In these tumours, we see an orchestrated, smouldering, tumourpromoting inflammation, with all kinds of leukocytes present (Figure 2). The predominant cells are CD8 + CTLs with a weaker presence of CD4 + cells and also CD3 + FoxP3 + Tregs. ${ }^{33}$ The number of infiltrating $\mathrm{CD} 163+\mathrm{M} 2$ macrophages is related to the number of Tregs, but it is not clear whether these cells interact. Different types of tumour-infiltrating leukocytes can affect each other's function. For example, myeloidderived suppressor cells may induce the maturation of CD4 + Tregs, which suppress the immune response. ${ }^{34}$ As with TAMs, the tumour-promoting functions of $\mathrm{T}$ lymphocytes are mediated by cytokines.

Two types of killer lymphocytes can be identified, the NK cells and the CD8 + T lymphocytes: CD8 + T cells are able to kill cancer cells, but only when a tumourspecific antigen is expressed together with a sufficient 

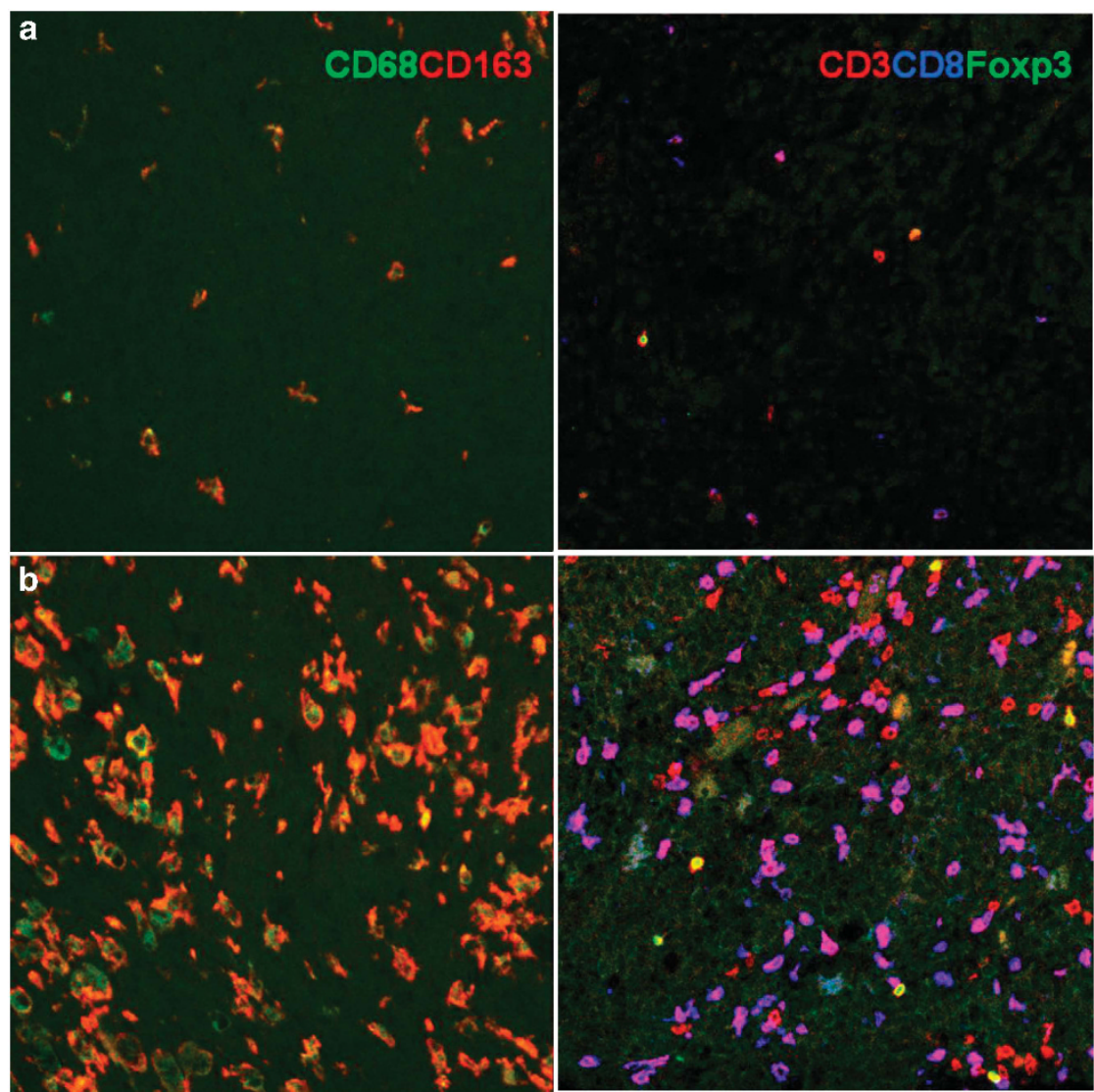

Figure 2 Subsets of intratumoral leukocytes. Immunofluorescence staining of macrophages in uveal melanoma using two antibodies directed against CD68 or CD163, and CD3, CD8, and Foxp3 to detect subtypes of lymphocytes. (a) A tumour with a low immune infiltrate and (b) a tumour with a high number of leukocytes.

expression of HLA class I antigens. On the other hand, NK cells eliminate tumours that lack HLA class I or that overexpress ligands for activating NK receptors. The ligand NKG2D (ie, MIC-A/B) is expressed by $50 \%$ of primary uveal melanomas but none of the metastases. ${ }^{35}$ Downregulation of HLA class I, which is a common mechanism for evading CD8 + cells, renders tumours more susceptible to NK cell-mediated lysis. The finding that loss of classical HLA class I molecules in uveal melanomas is associated with an improved prognosis suggests that NK cells act as the predominant cells responsible for immune surveillance of this tumour. ${ }^{36}$ Such immune surveillance may occur in the bloodstream, where NK cells seem able to kill migrating uveal melanoma cells before they reach the liver.

\section{Suppression of immune responses}

While uveal melanoma cells carrying tumour-specific antigens and tumour-infiltrating CD4 and CD8 cells are present, Tregs are also present in the primary tumour. These Tregs can suppress the activation, proliferation, and effector functions (such as cytokine production) of a wide range of other immune cells. In many studies,

Treg accumulation within tumours is a marker for poor clinical outcome. ${ }^{37}$ When studying uveal melanoma, two studies found no association between Treg numbers in uveal melanoma and survival, ${ }^{38,39}$ although the presence of Tregs has been associated with the extent of cyclooxygenase- 2 expression, a bad prognostic factor. We do not yet know whether these cells are present in metastases and contribute to local immunosuppression in the liver.

\section{Soluble mediators: cytokines and chemokines}

The cytokine and chemokine expression profiles of the tumour microenvironment may be more relevant than the specific immune cell content. Different cytokines can either promote or inhibit tumour development and progression, regardless of their source. ${ }^{40}$ TAMs are an important source of cytokines. The perpetuation of inflammation is largely achieved through positive feedback loops that induce chemokine synthesis in 
malignant and stromal cells, leading to further recruitment of inflammatory cells. ${ }^{3}$ Many different cytokines are present in the aqueous humour and vitreous fluid of eyes with uveal melanoma. ${ }^{41,42}$ Unlike the aqueous humour, which is continuously replenished, the gel in the vitreous chamber is more stagnant, representing a chronic inflammatory status. Some of the factors produced may lead to immune suppression. For example, TGF- $\beta$ secretion by tumour cells leads to inhibition of dendritic cell activation as well as direct inhibition of T-cell and NK cell function. Furthermore, TGF- $\beta$ production by tumour cells can convert effector T cells into Tregs. TGF- $\beta$ may be produced by the tumour cells or by the inflammatory cells. ${ }^{43}$ Other tumour cell products, such as IL-10, CSF-1, and chemokines (eg, CCL2), can modulate macrophages to become M2-like, cancer-promoting cells. That macrophages can stimulate tumour growth in the eye is a result, in part, of endogenously produced IL-1. In a murine model, IL-1RA-neutralizing antibodies inhibited the growth of the tumour, and modified the tumour stroma, reducing the myeloid suppressor cells and increasing M1 macrophage polarization. ${ }^{44}$

There is evidence that Tregs are recruited into tumours by CCL17 and CCL22. These chemokines have been shown to promote M2 polarization, and indeed are produced by M2 cells. ${ }^{34}$ CCL2 has been shown to have a role not only in attracting tumour-promoting macrophages but also in promoting their survival and M2 polarization. We recently observed that both CCL22 and CCL2 were expressed in freshly cultured uveal melanoma cells as well as in monocytes. ${ }^{33}$ Furthermore, we observed that increased numbers of TAMs and lymphocytes, as well as HLA expression, were all associated with chromosome 3 loss (Figure 3). There is evidence that chromosome 3 is associated with an increased leukocyte population, but the molecules involved have not yet been identified. There is much

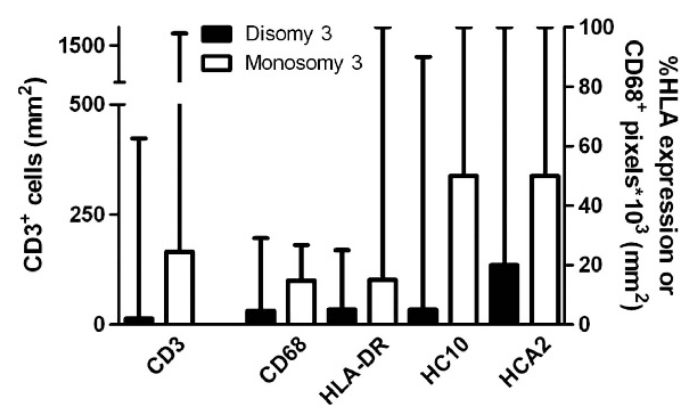

Figure 3 Significant associations between chromosome 3 status of 50 uveal melanoma and the inflammatory phenotype parameters. Median and range of the number of lymphocytes, macrophages, and the percentage of tumour HLA expression are displayed. scope for investigating this missing link, which is clearly of great importance in the inflammation-cancer connection. Do inflammatory chemokines and cytokines actually promote uveal melanomas or are they merely innocent epiphenomena?

\section{Impact of oxygen availability}

At some point, all solid malignancies outgrow their blood supply, becoming deprived of oxygen and nutrients. When cancer cells extend beyond the diffusion limits of nearby blood vessels, they metabolically adapt by preferentially undergoing glycolysis (even in the presence of oxygen). This escape mechanism not only provides a survival advantage over non-tranformed cells but also ensures that only the most successful cancer cells persist. ${ }^{45}$ As a consequence, oxygen-deficient (hypoxic) regions develop within the tumour. Local hypoxia stimulates cells to release proinflammatory mediators, recruiting inflammatory and immune cells, stimulating local angiogenesis, and providing surviving cancer cells with additional growth factors. ${ }^{46}$ Recruitment of leukocytes, including TAM, is largely dependent on mediators such as vascular endothelial growth factor. As most growing tumours contain some areas of hypoxia, it is not clear whether hypoxia is the direct driver of tumour angiogenesis or whether hypoxic stimuli generate inflammatory signals that drive angiogenesis. Indeed, Makitie et $a^{26}$ found an association between high levels of macrophages in uveal melanoma and a large tumour size, ${ }^{26}$ which subsequently could be confirmed. ${ }^{47}$ Exposing uveal melanoma cells in vitro to a hypoxic environment induced increased vascular endothelial growth factor production. ${ }^{48}$ There is no doubt that hypoxia has an important role in cancer; however, whether such hypoxia induces macrophage migration and differentiation in uveal melanoma is still not known.

\section{Immunotherapeutic options}

There are many strategies for cancer immunotherapy: therapeutic cancer vaccines, adoptive cell therapy, cytokine therapy, and antibody therapy. For uveal melanoma, an interesting approach is to develop a specific vaccine by transducing into tumour cells HLA class II genes as well as the gene for the immunostimulatory CD80 molecule. By preparing cells with the most common HLA class II genes, it is possible to prepare vaccines that should be effective in most patients. In vitro studies show that such uveal melanoma cells can specifically stimulate and later boost CD4+ T cells, as well as stimulating CD8 + cytotoxic cells. ${ }^{49}$

The introduction of CD80 into tumour cells has a secondary effect, which is to block the ligand PDL1, 
which usually inhibits antitumour T-cell activity. ${ }^{50}$ Another therapeutic approach, therefore, may be to block the interaction between ligand and receptor through antiPDL1 or anti-PD-1 monoclonal antibodies. This is currently being investigated in other cancers in many trials.

A molecule that is expressed at low levels on Tregs is cytotoxic T lymphocyte antigen 4 . To block the immune inhibitory effect of cytotoxic $\mathrm{T}$ lymphocyte antigen 4 binding to B7 on Tregs, patients receive infusions with anti-cytotoxic $\mathrm{T}$ lymphocyte antigen 4 monoclonal antibodies such as ipilimumab (Yervoy, Bristol-MyersSquibb, Princeton, NJ, USA). In patients with metastases of cutaneous melanoma, this drug has been noticed to improve survival. ${ }^{51,52}$ The effect of this drug with regard to uveal melanoma metastases has to be evaluated.

\section{Conclusions}

In uveal melanoma, an inflammatory phenotype that includes an increased presence of different types of lymphocytes and macrophages and an increased HLA class I and II expression is associated with a worse prognosis (loss of one chromosome 3 ), and may be related to the presence of hypoxia. Tregs have been identified in the tumour microenvironment, and may have a role in the absence of effective antitumour immune responses in patients with uveal melanoma. New therapeutic options are being developed that overcome the immunosuppressive effects, and are being found to be effective in the treatment of cutaneous melanoma metastases. Clinical trials are necessary to determine whether such new approaches will benefit the patients with uveal melanoma as well.

\section{Conflict of interest}

The authors declare no conflict of interest.

\section{Acknowledgements}

This work was financially supported by the Board of Directors of the Leiden University Medical Centre (PhD position recipient: IHGB), Stichting Nederlands Oogheelkundig Onderzoek, Rotterdamse Stichting Blindenbelangen, and Landelijke Stichting voor Blinden en Slechtzienden.

\section{References}

1 Colotta F, Allavena P, Sica A, Garlanda C, Mantovani A. Cancer-related inflammation, the seventh hallmark of cancer: links to genetic instability. Carcinogenesis 2009; 30 : 1073-1081.
2 Hanahan D, Weinberg RA. Hallmarks of cancer: the next generation. Cell 2011; 144: 646-674.

3 Mantovani A, Allavena P, Sica A, Balkwill F. Cancer-related inflammation. Nature 2008; 454: 436-444.

4 Flavell RA, Sanjabi S, Wrzesinski SH, Licona-Limon P. The polarization of immune cells in the tumour environment by TGFbeta. Nat Rev Immunol 2010; 10: 554-567.

5 Kilic E, Bruggenwirth HT, Verbiest MM, Zwarthoff EC, Mooy NM, Luyten GP et al. The RAS-BRAF kinase pathway is not involved in uveal melanoma. Melanoma Res 2004; 14: 203-205.

6 Tschentscher F, Husing J, Holter T, Kruse E, Dresen IG, Jöckel $\mathrm{KH}$ et al. Tumor classification based on gene expression profiling shows that uveal melanomas with and without monosomy 3 represent two distinct entities. Cancer Res 2003; 63: 2578-2584.

7 Van Raamsdonk CD, Bezrookove V, Green G, Bauer J, Gaugler L, O'Brien JM et al. Frequent somatic mutations of GNAQ in uveal melanoma and blue naevi. Nature 2009; 457: 599-602.

8 Van Raamsdonk CD, Griewank KG, Crosby MB, Garrido MC, Vemula S, Wiesner Tet al. Mutations in GNA11 in uveal melanoma. N Engl J Med 2010; 363: 2191-2199.

9 Zuidervaart W, van NF, Stark M, Dijkman R, Packer L, Borgstein AM et al. Activation of the MAPK pathway is a common event in uveal melanomas although it rarely occurs through mutation of BRAF or RAS. Br J Cancer 2005; 92: 2032-2038.

10 Bedikian AY, Legha SS, Mavligit G, Carrasco CH, Khorana $\mathrm{S}$, Plager $\mathrm{C}$ et al. Treatment of uveal melanoma metastatic to the liver: a review of the M. D. Anderson Cancer Center experience and prognostic factors. Cancer 1995; 76: $1665-1670$.

11 AJCC-UICC Ophthalmic Oncology Task Force. Malignant melanoma of the uvea. In: Edge SB, Byrd DR, Compton CC, Fritz AG, Greene FL, Trotti A (eds). Cancer Staging Manual. 7th edn. Springer: New York, 2010, pp 547-560.

12 Singh AD, Shields CL, Shields JA. Prognostic factors in uveal melanoma. Melanoma Res 2001; 11: 255-263.

13 Damato B, Eleuteri A, Taktak AF, Coupland SE. Estimating prognosis for survival after treatment of choroidal melanoma. Prog Retin Eye Res 2011; 30: 285-295.

14 Prescher G, Bornfeld N, Hirche H, Horsthemke B, Jockel $\mathrm{KH}$, Becher R. Prognostic implications of monosomy 3 in uveal melanoma. Lancet 1996; 347: 1222-1225.

15 Onken MD, Worley LA, Ehlers JP, Harbour JW. Gene expression profiling in uveal melanoma reveals two molecular classes and predicts metastatic death. Cancer Res 2004; 64: 7205-7209.

16 Fridman WH, Galon J, Pages F, Tartour E, Sautes-Fridman C, Kroemer G. Prognostic and predictive impact of intraand peritumoral immune infiltrates. Cancer Res 2011; 71: 5601-5605.

17 Mlecnik B, Bindea G, Pages F, Galon J. Tumor immunosurveillance in human cancers. Cancer Metast Rev 2011; 30: 5-12.

18 Maat W, Ly LV, Jordanova ES, Wolff-Rouendaal D, Schalij-Delfos NE, Jager MJ. Monosomy of chromosome 3 and an inflammatory phenotype occur together in uveal melanoma. Invest Ophthalmol Vis Sci 2008; 49: 505-510.

19 De Waard-Siebinga I, Hilders CG, Hansen BE, van Delft JL, Jager MJ. HLA expression and tumor-infiltrating immune cells in uveal melanoma. Graefes Arch Clin Exp Ophthalmol 1996; 234: 34-42. 
20 Bindea G, Mlecnik B, Fridman WH, Galon J. The prognostic impact of anti-cancer immune response: a novel classification of cancer patients. Semin Immunopathol 2011; 33: 335-340.

21 Streilein JW. Ocular immune privilege and the Faustian dilemma. The Proctor Lecture. Invest Ophthalmol Vis Sci 1996; 37: 1940-1950.

22 Dvorak HF. Tumors: wounds that do not heal. Similarities between tumor stroma generation and wound healing. N Engl J Med 1986; 315: 1650-1659.

23 van Kempen LC, Ruiter DJ, van Muijen GN, Coussens LM. The tumor microenvironment: a critical determinant of neoplastic evolution. Eur J Cell Biol 2003; 82: 539-548.

24 Coussens LM, Werb Z. Inflammation and cancer. Nature 2002; 420: 860-867.

25 Murdoch C, Muthana M, Coffelt SB, Lewis CE. The role of myeloid cells in the promotion of tumour angiogenesis. Nat Rev Cancer 2008; 8: 618-631.

26 Makitie T, Summanen P, Tarkkanen A, Kivela T. Tumor-infiltrating macrophages $(\mathrm{CD} 68(+)$ cells) and prognosis in malignant uveal melanoma. Invest Ophthalmol Vis Sci 2001; 42: 1414-1421.

27 Bronkhorst IH, Ly LV, Jordanova ES, Vrolijk J, Versluis M, Luyten GP et al. Detection of m2-macrophages in uveal melanoma and relation with survival. Invest Ophthalmol Vis Sci 2011; 52: 643-650.

28 Mantovani A, Germano G, Marchesi F, Locatelli M, Biswas SK. Cancer-promoting tumor-associated macrophages: new vistas and open questions. Eur J Immunol 2011; 41: 2522-2525.

29 Ly LV, Baghat A, Versluis M, Jordanova ES, Luyten GP, van Rooijen $\mathrm{N}$ et al. In aged mice, outgrowth of intraocular melanoma depends on proangiogenic M2-type macrophages. J Immunol 2010; 185: 3481-3488.

30 Toivonen P, Makitie T, Kujala E, Kivela T. Microcirculation and tumor-infiltrating macrophages in choroidal and ciliary body melanoma and corresponding metastases. Invest Ophthalmol Vis Sci 2004; 45: 1-6.

31 Smyth MJ, Dunn GP, Schreiber RD. Cancer immunosurveillance and immunoediting: the roles of immunity in suppressing tumor development and shaping tumor immunogenicity. Adv Immunol 2006; 90: 1-50.

32 Grivennikov SI, Greten FR, Karin M. Immunity, inflammation, and cancer. Cell 2010; 140: 883-899.

33 Bronkhorst IH, Vu TH, Jordanova ES, Luyten GP, Burg SH, Jager MJ. Different subsets of tumor-infiltrating lymphocytes correlate with macrophage influx and monosomy 3 in uveal melanoma. Invest Ophthalmol Vis Sci 2012; 53: 5370-5378.

34 Biswas SK, Mantovani A. Macrophage plasticity and interaction with lymphocyte subsets: cancer as a paradigm. Nat Immunol 2010; 11: 889-896.

35 Vetter CS, Lieb W, Brocker EB, Becker JC. Loss of nonclassical MHC molecules MIC-A/B expression during progression of uveal melanoma. Br J Cancer 2004; 91: 1495-1499.
36 Blom DJ, Luyten GP, Mooy C, Kerkvliet S, Zwinderman AH, Jager MJ. Human leukocyte antigen class I expression. Marker of poor prognosis in uveal melanoma. Invest Ophthalmol Vis Sci 1997; 38: 1865-1872.

37 Quezada SA, Peggs KS, Simpson TR, Allison JP. Shifting the equilibrium in cancer immunoediting: from tumor tolerance to eradication. Immunol Rev 2011; 241: 104-118.

38 Lagouros E, Salomao D, Thorland E, Hodge DO, Vile R, Pulido JS. Infiltrative T regulatory cells in enucleated uveal melanomas. Trans Am Ophthalmol Soc 2009; 107: 223-228.

39 Mougiakakos D, Johansson CC, Trocme E, All-Ericsson C, Economou MA, Larsson $\mathrm{O}$ et al. Intratumoral forkhead box P3-positive regulatory $\mathrm{T}$ cells predict poor survival in cyclooxygenase-2-positive uveal melanoma. Cancer 2010; 116: 2224-2233.

40 Lin WW, Karin M. A cytokine-mediated link between innate immunity, inflammation, and cancer. J Clin Invest 2007; 117: 1175-1183.

41 Ly LV, Bronkhorst IH, van Beelen E, Vrolijk J, Taylor AW, Versluis $\mathrm{M}$ et al. Inflammatory cytokines in eyes with uveal melanoma and relation with macrophage infiltration. Invest Ophthalmol Vis Sci 2010; 51: 5445-5451.

42 Nagarkatti-Gude N, Bronkhorst IH, van Duinen SG, Luyten GP, Jager MJ. Cytokines and chemokines in the vitreous fluid of eyes with uveal melanoma. Invest Ophthalmol Vis Sci 2012; 53: 6748-6755.

43 Esser P, Grisanti S, Bartz-Schmidt K. TGF-beta in uveal melanoma. Microsc Res Technol 2001; 52: 396-400.

44 Triozzi PL, Aldrich W, Singh A. Effects of interleukin-1 receptor antagonist on tumor stroma in experimental uveal melanoma. Invest Ophthalmol Vis Sci 2011; 52: 5529-5535.

45 Gatenby RA, Gillies RJ. Why do cancers have high aerobic glycolysis? Nat Rev Cancer 2004; 4: 891-899

46 Karin M. NF-kappaB and cancer: mechanisms and targets. Mol Carcinogen 2006; 45: 355-361.

47 Jager MJ, Ly LV, El Filali M, Madigan MC. Macrophages in uveal melanoma and in experimental ocular tumor models: Friends or foes? Prog Retin Eye Res 2011; 30: 129-146

48 El Filali M, Missotten GS, Maat W, Ly LV, Luyten GP, van der Velden PA et al. Regulation of VEGF-A in uveal melanoma. Invest Ophthalmol Vis Sci 2010; 51: 2329-2337.

49 Bosch JJ. Activation of tumor-specific T cells by MHC II uveal melanoma vaccines, PhD thesis, Leiden University Medical Center: Leiden, The Netherlands, 2012.

50 Haile ST, Bosch JJ, Agu NI, Zeender AM, Somasundaram P, Srivastava MK et al. Tumor cell programmed death ligand 1-mediated $\mathrm{T}$ cell suppression is overcome by coexpression of CD80. J Immunol 2011; 186: 6822-6829.

51 Hodi FS, O'Day SJ, McDermott DF, Weber RW, Sosman JA, Haanen JB et al. Improved survival with ipilimumab in patients with metastatic melanoma. N Engl J Med 2010; 363: 711-723.

52 Robert C, Thomas L, Bondarenko I, O'Day S, Weber J, Garbe $\mathrm{C}$ et al. Ipilimumab plus dacarbazine for previously untreated metastatic melanoma. N Engl J Med 2011; 364: 2517-2526. 